\title{
Characterization and Thermal Activation of Adducts of Group 4
}

\author{
Tetrahalides with 1,2-Dialkoxyalkanes
}

\author{
Fabio Marchetti ${ }^{\S}$ and Guido Pampaloni *
}

Università di Pisa, Dipartimento di Chimica e Chimica Industriale, Via Risorgimento 35, I-56126 Pisa, Italy

Received......; accepted

Corresponding author. Tel.: int. code + 0502219 219; fax: int. code + 0502219246 .

E-mail address:pampa@dcci.unipi.it.

${ }^{\S}$ Born in Bologna in 1974. 


\begin{abstract}
Coordination compounds of general formula $\mathrm{MX}_{4}\left[\kappa^{2}-\mathrm{O}(\mathrm{R}) \mathrm{CH}_{2} \mathrm{CH}\left(\mathrm{R}^{\prime}\right) \mathrm{OR}^{\prime \prime}\right]$, 2a-i, have been prepared in high yield upon addition of a variety of 1,2-dialkoxyalkanes, $\mathrm{ROCH}_{2} \mathrm{CH}\left(\mathrm{R}^{\prime}\right) \mathrm{OR}^{\prime \prime}$, to $\mathrm{MX}_{4}(\mathrm{M}$ $=\mathrm{Ti}, \mathrm{X}=\mathrm{F}, \mathbf{1 a}, \mathrm{I}, \mathbf{1 b} ; \mathrm{M}=\mathrm{Zr}, \mathrm{X}=\mathrm{Br}, \mathbf{1 c}, \mathrm{I}, \mathbf{1 d})$. The new complexes have been characterized by elemental analysis and NMR spectroscopy. The thermal stability of $\mathbf{2}$ has been studied. Fragmentation of the $\mathrm{TiI}_{4}$ adducts takes place in chloroform solution at $90^{\circ} \mathrm{C}$ : after hydrolysis of the reaction mixtures, MeI and $\mathrm{CH}_{2} \mathrm{ICH}_{2} \mathrm{I}$ have been detected from $\mathrm{TiI}_{4}[\mathrm{dme}]\left[\mathrm{dme}=\mathrm{MeO}\left(\mathrm{CH}_{2}\right)_{2} \mathrm{OMe}\right], \mathrm{MeI}$ and $\mathrm{Me}_{2} \mathrm{O}$ from $\mathrm{TiI}_{4}\left[\mathrm{MeOCH}_{2} \mathrm{CH}(\mathrm{Me}) \mathrm{OMe}\right]$, and $\mathrm{MeI}, \mathrm{CH}_{2} \mathrm{ICH}_{2} \mathrm{I}, \mathrm{Me}_{2} \mathrm{O}, \mathrm{CH}_{2} \mathrm{ClI}$ from $\mathrm{TiI}_{4}\left[\mathrm{MeO}\left(\mathrm{CH}_{2}\right)_{2} \mathrm{OCH}_{2} \mathrm{Cl}\right]$. The diether $\mathrm{MeO}\left(\mathrm{CH}_{2}\right)_{2} \mathrm{OCH}_{2} \mathrm{Cl}$ is thermally activated also when coordinated to $\mathrm{ZrX} 4(\mathrm{X}=\mathrm{Br}$, I): after hydrolysis, $\mathrm{MeBr}, \mathrm{CH}_{2} \mathrm{BrCH}_{2} \mathrm{Br}, \mathrm{O}\left(\mathrm{CH}_{2} \mathrm{CH}_{2} \mathrm{Cl}\right)_{2}$ and $\mathrm{Me}_{2} \mathrm{O}(\mathrm{X}=\mathrm{Br})$ and $\mathrm{MeI}, \mathrm{CH}_{2} \mathrm{ClI}$ and $\mathrm{CH}_{2} \mathrm{ICH}_{2} \mathrm{I}(\mathrm{X}$ = I) have been found respectively.
\end{abstract}

Keywords: Titanium, zirconium, 1,2-dimethoxyethane, thermal stability, $\mathrm{C}-\mathrm{O}$ bond cleavage

\title{
1. Introduction
}

Early transition metal halides have been increasingly used as promoters of synthetic processes, and the alkene polymerization reactions promoted by derivatives of Group 4 metal halides have definitely became a fundamental issue of the modern organometallic chemistry [1]. Recent examples of relevant organic reactions mediated by the homoleptic halides of titanium,[2] zirconium [3] and hafnium [4] are given. 
In the very last years, we have been involved in the study of the reactivity of niobium and tantalum pentahalides with molecules containing oxygen as donor atom, since this field of chemistry looked scarcely developed [5]. We have shown that the reactions of $\mathrm{MX}_{5}$ with $O$-donors can lead to simple coordination adducts which, under appropriate conditions, may evolve as result of $\mathrm{C}-\mathrm{Y}$ bond activation $(\mathrm{Y}=\mathrm{H}, \mathrm{O})[5 \mathrm{a}-\mathrm{b}, 6]$. Remarkably, we have reported that the reaction of $\mathrm{NbCl}_{5}$ with 1,2 dimethoxyethane (dme) proceeds selectively at room temperature with formation of 1,4-dioxane as result of multiple breaking and coupling of $\mathrm{C}-\mathrm{O}$ bonds [6d]. Further studies have indicated that alternative transformations may be operative for different 1,2-dialkoxyalkanes when reacted with $\mathrm{MCl}_{5}$ $(\mathrm{M}=\mathrm{Nb}, \mathrm{Ta})[6 \mathrm{e}]$.

The reactions of $\mathrm{MX}_{5}(\mathrm{M}=\mathrm{Nb}, \mathrm{Ta})$ with 1,2-dialkoxyalkanes are strongly influenced by the nature of the halide: thus, the fluorides $\mathrm{MF}_{5}$ activate the organic substrate at high temperature only, giving unusual fragmentations which do not affect the $\left[\mathrm{MF}_{5}\right]$ frame, as consequence of the relatively high $\mathrm{M}-\mathrm{F}$ bond energy [6a]. This feature has suggested that the use of homoleptic early transition metal fluorides in catalytic processes involving oxygen compounds would deserve more attention.

For what concerns Group 4 tetrahalides, $\mathbf{1}$, it has been known since long time that $\mathrm{MCl}_{4}(\mathrm{M}=\mathrm{Ti}$, $\mathrm{Zr}$, Hf) form stable hexacoordinated adducts with dme, i.e. $\mathrm{MCl}_{4}(\mathrm{dme})$ [7]. However, this piece of chemistry has been only limitedly extended to the other halides, i.e. fluorides, bromides and iodides $[7 \mathrm{~d}, \mathrm{e}, 8]$. Moreover, the possibility to activate the bidentate $O$-ligand by thermal treatment has not been explored so far.

On account of the noticeable chemistry exhibited by Group 5 pentahalides with dme and 1,2dialkoxyalkanes in general, we moved to put some more light into the analogous reactivity of the Group 4 tetrahalides. Thus, we have prepared a series of new coordination compounds of $\mathrm{MX}_{4}(\mathrm{M}=\mathrm{Ti}, \mathrm{X}=\mathrm{F}$, 1a, I, 1b; M = Zr, X = Br, 1c, I, 1d) with 1,2-dialkoxyalkanes (dme, 1,2-dimethoxypropane, 2- 
methoxyethoxymethyl chloride). The new compounds have been characterized by NMR in solution. Moreover, their thermal stability has been investigated. The clear detection of the organic fragments originated from the activation reactions has been possible by hydrolysis of the reaction mixtures, thus making the $O$-containing species free from coordination to the metal centres [9].

\section{Results and Discussion}

The complexes $\mathrm{MX}_{4}\left[\kappa^{2}-\mathrm{O}(\mathrm{R}) \mathrm{CH}_{2} \mathrm{CH}\left(\mathrm{R}^{\prime}\right) \mathrm{OR}^{\prime \prime}\right]$, 2a-i, have been obtained in high yields upon addition of 1,2-dialkoxyalkanes to Group 4 tetrahalides, see Scheme 1.

\section{Scheme 1 about here}

Compounds 2a-i, resembling the known reported complexes $\mathrm{MX}_{4}(\mathrm{dme})(\mathrm{M}=\mathrm{Ti}, \mathrm{X}=\mathrm{Cl}, \mathrm{Br} ; \mathrm{M}=$ $\mathrm{Zr}, \mathrm{X}=\mathrm{Cl}$ ) [7] have been characterized by spectroscopic and analytical techniques. According to the data, the diether ligands act as bidentate in mononuclear species. Indeed the NMR spectra exhibit the resonances due to symmetrically coordinated organic frames, at chemical shifts generally higher than those found in the respective uncoordinated molecules [e.g. for $\mathrm{MeO}\left(\mathrm{CH}_{2}\right)_{2} \mathrm{OCH}_{2} \mathrm{Cl}$ in $\mathbf{2 h}: \delta\left({ }^{1} \mathrm{H}\right)=5.82$ $\left(\mathrm{CH} \mathrm{Cl}_{2} \mathrm{Cl}\right), 4.18,3.88\left(\mathrm{CH}_{2} \mathrm{CH}_{2}\right), 3.88$ ppm $(\mathrm{OMe}) ; \delta\left({ }^{13} \mathrm{C}\right): 78.6\left(\mathrm{CH}_{2} \mathrm{Cl}\right), 76.0,70.8\left(\mathrm{CH}_{2} \mathrm{CH}_{2}\right), 64.8 \mathrm{ppm}$ $(\mathrm{OMe})$. For uncoordinated 2-methoxyethoxymethyl chloride: $\delta\left({ }^{1} \mathrm{H}\right)=5.54\left(\mathrm{CH}_{2} \mathrm{Cl}\right), 3.83,3.60$ $\left(\mathrm{CH}_{2} \mathrm{CH}_{2}\right), 3.39$ ppm $(\mathrm{OMe}) ; \delta\left({ }^{13} \mathrm{C}\right): 83.2\left(\mathrm{CH}_{2} \mathrm{Cl}\right), 71.0,69.4\left(\mathrm{CH}_{2} \mathrm{CH}_{2}\right), 59.0$ ppm $\left.(\mathrm{OMe})\right]$. Moreover, solution electrical conductivity values are comparable with those typical of neutral early-transition halide derivatives [6b,d], accounting for the monomeric structure of $\mathbf{2 a - i}$. 
At variance with Group 5 pentahalides [6d], the reactions of 1 with 1,2-dialkoxyalkanes give the same outcome even when the oxygen compound is used in excess: indeed compounds $\mathbf{2 a - i}$ are generated in high yields also by using a three-fold molar excess of organic material with respect to the metal species, neither complexes containing more than one 1,2-dialkoxyalkane ligand have been obtained.

Compounds 2 a-i are stable at room temperature, both in the solid state and in chlorinated solvents. In order to investigate the possibility to activate the organic moiety, we tested the thermal stability of these complexes by heating $\mathrm{CDCl}_{3}$ solutions of $\mathbf{2 a - i}$ in sealed NMR tubes at $c a .90^{\circ} \mathrm{C}$ (temperature of the external oil-bath). The resulting mixtures consisted of products which could not be identified, thus excess of water was added causing the precipitation of almost colourless solids (probably metal oxides). The composition of the organic layer was determined by GC-MS and NMR spectroscopy.

The analyses pointed out the presence of $\mathrm{MeO}\left(\mathrm{CH}_{2}\right)_{2} \mathrm{OMe}$ in the solutions deriving from $\mathbf{2 a , c , d}$, and of $\mathrm{MeOCH}_{2} \mathrm{CH}(\mathrm{Me}) \mathrm{OMe}$ in the solution obtained from $\mathbf{2 f}$, indicating that the parent compounds did not undergo thermal degradation. Differently, fragmentation of the 1,2-dialkoxyalkanes takes place at high temperature within the iodo-complexes $\operatorname{TiI}_{4}\left(\kappa^{2}-\mathrm{dme}\right), \mathbf{2 b}$, and $\mathrm{TiI}_{4}\left[\kappa^{2}-\mathrm{O}(\mathrm{Me}) \mathrm{CH}_{2} \mathrm{CH}(\mathrm{Me}) \mathrm{OMe}\right], \mathbf{2 e}$. More in detail, $\mathrm{MeI} / \mathrm{CH}_{2} \mathrm{ICH}_{2} \mathrm{I}$ and $\mathrm{MeI} / \mathrm{Me}_{2} \mathrm{O}$ have been detected as main components of the solutions obtained by thermal treatment of $\mathbf{2 b}$ and $\mathbf{2 e}$, respectively. The fragmentation of $\mathbf{2 e}$ is not selective, since unidentified compounds form together with $\mathrm{MeI}$ and $\mathrm{Me}_{2} \mathrm{O}$. The fragmentation reactions proceed clearly via the activation of $\mathrm{Ti}-\mathrm{I}$ and $\mathrm{C}-\mathrm{O}$ bonds, and the formation of new $\mathrm{C}-\mathrm{I}$ bonds.

Our experiments have confirmed that $\mathrm{MeO}\left(\mathrm{CH}_{2}\right)_{2} \mathrm{OCH}_{2} \mathrm{Cl}$ is more reactive than the congener molecules [6e], it undergoes quick activation at high temperature when coordinated in the complexes $\mathbf{2 g - i}$. Hence, MeI, $\mathrm{CH}_{2} \mathrm{ICH}_{2} \mathrm{I}$ and $\mathrm{CH}_{2} \mathrm{ClI}$ have been identified as organic products of the degradation processes of $\mathbf{2 g}$, $\mathbf{i}$ after hydrolysis, suggesting that several pathways are operative, involving the cleavage of $\mathrm{M}-\mathrm{I}$ and $\mathrm{C}-\mathrm{O}$ bonds. Interestingly, dimethyl ether has been found as further product of the fragmentation of $\mathbf{2 g}$, 
indicating that $\mathrm{C}-\mathrm{O}$ coupling is effective in this case to some extent. The degradation of $\mathrm{MeO}\left(\mathrm{CH}_{2}\right)_{2} \mathrm{OCH}_{2} \mathrm{Cl}$ by $\mathrm{ZrBr}_{4}$ is even less selective, leading to $\mathrm{MeBr}, \mathrm{CH}_{2} \mathrm{BrCH}_{2} \mathrm{Br}, \mathrm{O}\left(\mathrm{CH}_{2} \mathrm{CH}_{2} \mathrm{Cl}\right)_{2}$ and $\mathrm{Me}_{2} \mathrm{O}$. The products of the fragmentation reactions promoted by Group 4 halides are summarized in table 1.

\section{Table 1 about here}

Usually, the direct combination of ethers with oxophilic metal complexes (lanthanides, early transition elements) generally proceeds with $\mathrm{C}-\mathrm{O}$ bond cleavage and formation of stable metal-alkoxides [10]. In other words, the starting ether does not transform into functionalized species, but breaks down into smaller fragments. For what concerns 1,2-dimethoxyethane, dme, it has been reported that this can react with transition metal compounds undergoing cleavage of one or two $\mathrm{O}-\mathrm{CH}_{2}$ bonds [11], to afford methoxy-derivatives and, in some cases, free or coordinated ethylene [11e]. Otherwise, the cleavage of the dme $\mathrm{O}-\mathrm{CH}_{3}$ bonds can be performed by aluminum derivatives in mild conditions [12].

In agreement with these considerations, the thermal treatment of $\mathbf{2} \mathbf{b}, \mathbf{e}, \mathbf{g}, \mathbf{h}, \mathbf{i}$ may afford some metalalkoxides, in admixture with organic molecules. The NMR characterization of these mixtures has failed, thus the successive addition of water has been required for the detection of the fragments (see above). The hydrolysis of $\mathrm{MX}_{4}$ derivatives $(\mathrm{M}=\mathrm{Ti}, \mathrm{Zr}, \mathrm{X}=$ halogen) takes place with releasing of $\mathrm{HX}$, therefore during this process the alkoxide moieties, $\mathrm{M}-\mathrm{OR}$, should be converted into halide species, $\mathrm{X}-\mathrm{R}$, and these can be recognized finally. Similar features have been found regarding $\mathrm{MX}_{5}(M=N b, T a, X=$ halogen) derivatives [6].

\section{Conclusions}


Group 4 tetrahalides form hexacoordinated adducts upon addition of 1,2-dialkoxyalkanes. The coordination compounds are stable at room temperature, but they may undergo thermal activation in chlorinated solvents. The activation, favoured by low metal-halide bond energy, is not selective, and does not generally include the formation of new $\mathrm{C}-\mathrm{O}$ bonds by coupling of the resulting fragments. This feature is in accordance with the common ether fragmentation routes by means of transition metal derivatives [10-12], and contrasts with recent findings concerning Group 5 pentahalides, which are capable of activating 1,2-dialkoxyalkanes via unusual pathways including $\mathrm{C}-\mathrm{O}$ bond formation.

\section{Experimental}

All manipulations of air and/or moisture sensitive compounds were performed under atmosphere of pre-purified Argon using standard Schlenk techniques. The reaction vessels were oven dried at 150 ${ }^{\circ} \mathrm{C}$ prior to use, evacuated $\left(10^{-2} \mathrm{mmHg}\right)$ and then filled with argon. $\mathrm{MX}_{4}(\mathrm{M}=\mathrm{Ti}, \mathrm{X}=\mathrm{F}, \mathbf{1 a}, \mathrm{I}, \mathbf{1 b} ; \mathrm{M}=$ $\mathrm{Zr}, \mathrm{X}=\mathrm{Br}, \mathbf{1 c}, \mathrm{I}, \mathbf{1 d})$ were commercial products (Aldrich) of the highest purity available, stored under argon atmosphere as received. $\mathrm{CH}_{2} \mathrm{Cl}_{2}, \mathrm{CHCl}_{3}, \mathrm{CD}_{2} \mathrm{Cl}_{2}, \mathrm{CDCl}_{3}, \mathrm{MeO}\left(\mathrm{CH}_{2}\right)_{2} \mathrm{OMe}$ (dme) $\mathrm{MeOCH}_{2} \mathrm{CH}(\mathrm{Me}) \mathrm{OMe}$, and $\mathrm{MeOCH}_{2} \mathrm{CH}_{2} \mathrm{OCH}_{2} \mathrm{Cl}$ were distilled before use under argon atmosphere from $\mathrm{P}_{4} \mathrm{O}_{10}$, while pentane was distilled from $\mathrm{LiAlH}_{4}$. Infrared spectra were recorded at $298 \mathrm{~K}$ on a FT IR-Perkin Elmer Spectrometer, equipped with a UATR sampling accessory. NMR measurements were recorded on Varian Gemini 200BB at $298 \mathrm{~K}$, unless otherwise specified. The chemical shifts for ${ }^{1} \mathrm{H}$ and ${ }^{13} \mathrm{C}$ were referenced to the non-deuterated aliquot of the solvent. GC/MS analyses were performed on a HP6890 instrument, interfaced with MSD-HP5973 detector and equipped with a Phenonex Zebron column. Molar conductivities $\left(\Lambda_{\mathrm{M}}\right)$ were calculated per mole of metal atoms on the basis of resistance measurements performed by a Metrohm AG Konduktometer E382 Instrument (cell constant $=0.815$ $\mathrm{cm}^{\square 1}$ ) on dichloromethane solutions $c a .0 .010 \mathrm{M}$, at $293 \mathrm{~K}$ [13]. Carbon and hydrogen analyses were 
performed on a Carlo Erba mod. 1106 instrument, paying particular attention to the more sensitive compounds which were weighed and directly introduced into the analyzer. The halide (bromide or iodide) content was determined by the Volhardt method [14] after exhaustive hydrolysis of the sample. The metal was analyzed as $\mathrm{MO}_{2}(\mathrm{M}=\mathrm{Ti}, \mathrm{Zr})$, obtained by hydrolysis of the sample followed by calcination in a platinum crucible. The halogen and the metal analyses were repeated twice in order to check for reproducibility.

4.1. Preparation and thermal stability of $M X_{4}\left[\kappa^{2}-O(R) C H_{2} C H(R) O R^{\prime \prime}\right]\left(M=T i, X=F, R=R^{\prime \prime}=M e\right.$, $R^{\prime}=H, 2 \boldsymbol{a} ; M=T i, X=I, R=R^{\prime \prime}=M e, R^{\prime}=H, 2 \boldsymbol{b} ; M=Z r, X=B r, R=R^{\prime \prime}=M e, R^{\prime}=H, 2 \boldsymbol{c} ; M=$ $Z r, X=I, R=R^{\prime \prime}=M e, R^{\prime}=H, 2 d ; M=T i, X=I, R=R^{\prime}=R^{\prime \prime}=M e, 2 \boldsymbol{e} ; M=Z r, X=B r, R=R^{\prime}=R^{\prime \prime}$ $=M e, 2 f ; M=T i, X=I, R=M e, R^{\prime}=H, R^{\prime \prime}=C H_{2} C l, 2 g ; M=Z r, X=B r, R=M e, R^{\prime}=H, R^{\prime \prime}=$ $\left.\mathrm{CH}_{2} \mathrm{Cl}, 2 \boldsymbol{h} ; \mathrm{M}=\mathrm{Zr}, \mathrm{X}=\mathrm{I}, \mathrm{R}=\mathrm{Me}, \mathrm{R}^{\prime}=\mathrm{H}, \mathrm{R}^{\prime \prime}=\mathrm{CH}_{2} \mathrm{Cl}, 2 \boldsymbol{i}\right)$.

The preparation of $\mathbf{2 a}$ is described in detail; compounds $\mathbf{2} \mathbf{b}-\mathbf{i}$ were obtained by analogous procedure. Titanium tetrafluoride $(0.080 \mathrm{~g}, 0.65 \mathrm{mmol})$ in $\mathrm{CH}_{2} \mathrm{Cl}_{2}(10 \mathrm{~mL})$ was treated with dme $(0.070$ $\mathrm{mL}, 0.67 \mathrm{mmol}$ ), and the resulting mixture was stirred for 4 hours at room temperature. The product $2 \mathrm{a}$ was then obtained as a colorless solid by removal of the volatile materials under vacuo. Yield: $0.111 \mathrm{~g}$ (80\%). Anal. Calcd for $\mathrm{C}_{4} \mathrm{H}_{10} \mathrm{~F}_{4} \mathrm{O}_{2}$ Ti: C, 22.45; H, 4.71; Ti, 22.37. Found: C, 22.32; H, 4.58; Ti, 22.25 . ${ }^{1} \mathrm{H}$ NMR $\left.\left(\mathrm{CDCl}_{3}\right): \delta=3.70\left(\mathrm{~s}, 4 \mathrm{H}, \mathrm{CH} \mathrm{H}_{2}\right), 3.53 \mathrm{ppm}(\mathrm{s}, 6 \mathrm{H}, \mathrm{CH})_{3}\right) \cdot{ }^{13} \mathrm{C}\left\{{ }^{1} \mathrm{H}\right\} \mathrm{NMR}\left(\mathrm{CDCl}_{3}\right): \delta=72.2$ $\left(\mathrm{CH}_{2}\right), 60.6 \mathrm{ppm}\left(\mathrm{CH}_{3}\right) \cdot \Lambda_{M}=0.050 \mathrm{~S} \cdot \mathrm{cm}^{2} \cdot \mathrm{mol}^{-1}$.

In a different experiment, a solution of $\mathbf{2 a}(0.090 \mathrm{~g}, 0.42 \mathrm{mmol})$ in $\mathrm{CDCl}_{3}(0.85 \mathrm{~mL}) / \mathrm{CH}_{2} \mathrm{Cl}_{2}$ (0.420 mmol) was introduced into a NMR tube, then the tube was sealed and heated at $90^{\circ} \mathrm{C}$ (temperature of the external oil bath) for 2 hours. Afterwards, the tube was allowed to cool to room 
temperature, then it was opened and water $(c a .10 \mathrm{mmol})$ was added to the mixture. A dark precipitate formed, and this was separated from a light yellow solution. Combined NMR and GC-MS analyses on the latter revealed the presence of $\mathrm{CH}_{2} \mathrm{Cl}_{2}$ and dme in about 1:1 ratio.

$\mathrm{TiI}_{4}\left[\mathrm{\kappa}^{2}-\mathrm{O}(\mathrm{Me}) \mathrm{CH}_{2} \mathrm{CH}_{2} \mathrm{OMe}\right]$, 2b. Scarlet solid, $86 \%$ yield from $\mathbf{1 b}(0.245 \mathrm{~g}, 0.441 \mathrm{mmol})$ and dme (0.050 mL, $0.48 \mathrm{mmol})$. Time: 12 hours. Anal. Calcd for $\mathrm{C}_{4} \mathrm{H}_{10} \mathrm{I}_{4} \mathrm{O}_{2} \mathrm{Ti}: \mathrm{C}, 7.44 ; \mathrm{H}, 1.56$; Ti, 7.41; I, 78.63. Found: C, 7.32; H, 1.66; Ti, 7.26; I, 77.90. ${ }^{1} \mathrm{H} \mathrm{NMR}\left(\mathrm{CDCl}_{3}\right): \delta=4.59\left(\mathrm{~s}, 4 \mathrm{H}, \mathrm{CH}_{2}\right), 4.15 \mathrm{ppm}$ (s, $\left.6 \mathrm{H}, \mathrm{CH}_{3}\right) . \Lambda_{M}=0.28 \mathrm{~S} \cdot \mathrm{cm}^{2} \mathrm{~mol}^{-1}$. Thermal treatment / hydrolysis (GC/MS-NMR): $\mathrm{CH}_{2} \mathrm{Cl}_{2}, \mathrm{MeI}$, $\mathrm{CH}_{2} \mathrm{ICH}_{2} \mathrm{I}$ (ratio $\left.1: 2: 1\right)$.

$\mathrm{ZrBr}_{4}\left[\kappa^{2}-\mathrm{O}(\mathrm{Me}) \mathrm{CH}_{2} \mathrm{CH}_{2} \mathrm{OMe}\right], 2 \mathrm{c}$. Colourless solid, $90 \%$ yield from $1 \mathrm{c}(0.200 \mathrm{~g}, 0.487 \mathrm{mmol})$ and dme (0.055 mL, $0.53 \mathrm{mmol})$. Time: 2 hours. Anal. Calcd for $\mathrm{C}_{4} \mathrm{H}_{10} \mathrm{Br}_{4} \mathrm{O}_{2} \mathrm{Zr}$ : C, 9.59; H, 2.01; Zr, 18.21; $\mathrm{Br}$, 63.80. Found: C, 9.64; H, 1.95; Zr, 18.10; $\mathrm{Br}, 62.87 .{ }^{1} \mathrm{H}$ NMR $\left(\mathrm{CDCl}_{3}\right): \delta=4.55(\mathrm{~s}, 4 \mathrm{H}, \mathrm{CH}), 4.38$ $\operatorname{ppm}\left(\mathrm{s}, 6 \mathrm{H}, \mathrm{CH} \mathrm{H}_{3}\right) \cdot{ }^{13} \mathrm{C}\left\{{ }^{1} \mathrm{H}\right\} \mathrm{NMR}\left(\mathrm{CDCl}_{3}\right): \delta=76.0\left(\mathrm{CH}_{2}\right), 70.8 \mathrm{ppm}\left(\mathrm{CH}_{3}\right) . \Lambda_{M}=0.13 \mathrm{~S} \cdot \mathrm{cm}^{2} \cdot \mathrm{mol}^{-1}$. Thermal treatment / hydrolysis (GC/MS-NMR): $\mathrm{CH}_{2} \mathrm{Cl}_{2}$, dme (ratio 1:1).

$\mathrm{ZrI}_{4}\left[\kappa^{2}-\mathrm{O}(\mathrm{Me}) \mathrm{CH}_{2} \mathrm{CH}_{2} \mathrm{OMe}\right], 2 \mathrm{~d}$. Red solid, $80 \%$ yield from $\mathbf{1 d}(0.240 \mathrm{~g}, 0.401 \mathrm{mmol})$ and dme $(0.045$ mL, 0.43 mmol). Time: 4 hours. Anal. Calcd for $\mathrm{C}_{4} \mathrm{H}_{10} \mathrm{I}_{4} \mathrm{O}_{2} \mathrm{Zr}$ : C, 6.97; H, 1.46; Zr, 13.24; I, 73.68. Found: C, 6.89; H, 1.39; Zr, 13.12; I, 73.40. ${ }^{1} \mathrm{H} \mathrm{NMR}\left(\mathrm{CDCl}_{3}\right): \delta=4.45$ (s, $\left.4 \mathrm{H}, \mathrm{CH}_{2}\right), 4.29 \mathrm{ppm}(\mathrm{s}, 6 \mathrm{H}$, $\left.\mathrm{CH}_{3}\right) \cdot \Lambda_{\mathrm{M}}=0.10 \mathrm{~S} \cdot \mathrm{cm}^{2} \cdot \mathrm{mol}^{-1}$. Thermal treatment / hydrolysis (GC/MS-NMR): $\mathrm{CH}_{2} \mathrm{Cl}_{2}$, dme (ratio 1:1). $\mathrm{TiI}_{4}\left[\kappa^{2}-\mathrm{O}(\mathrm{Me}) \mathrm{CH}(\mathrm{Me}) \mathrm{CH}_{2} \mathrm{OMe}\right]$, 2e. Dark red solid, 79\% yield from $\mathbf{1 b}(0.220 \mathrm{~g}, 0.396 \mathrm{mmol})$ and 1,2dimethoxypropane $(0.050 \mathrm{~mL}, 0.41 \mathrm{mmol})$. Time: 12 hours. Anal. Calcd for $\mathrm{C}_{5} \mathrm{H}_{12} \mathrm{I}_{4} \mathrm{O}_{2} \mathrm{Ti}: \mathrm{C}, 9.10 ; \mathrm{H}$, 1.83; Ti, 7.26; I, 76.95. Found: C, 9.21; H, 1.72; Ti, 7.15; I, 74.99. ${ }^{1} \mathrm{H}$ NMR $\left(\mathrm{CDCl}_{3}\right): \delta=4.5$ (m-br, 3 $\mathrm{H}, \mathrm{CH}_{2} \mathrm{CH}$ ), 4.10 (s-br, $6 \mathrm{H}, \mathrm{OMe}$ ), 1.48 ppm (br, $\left.3 \mathrm{H}, \mathrm{CHMe}\right) .{ }^{13} \mathrm{C} \mathrm{NMR}\left(\mathrm{CDCl}_{3}\right): \delta=79.9,79.6$ 
$\left(\mathrm{CH}_{2} \mathrm{CH}\right), 66.8,64.7(\mathrm{OMe}), 13.8 \mathrm{ppm}(\mathrm{CHMe}) . \Lambda_{\mathrm{M}}=0.20 \mathrm{~S} \cdot \mathrm{cm}^{2} \mathrm{~mol}^{-1}$. Thermal treatment $/$ hydrolysis (GC/MS-NMR): $\mathrm{CH}_{2} \mathrm{Cl}_{2}, \mathrm{MeI}, \mathrm{Me}_{2} \mathrm{O}$ (ratio 2:3:1).

$\mathrm{ZrBr}_{4}\left[\kappa^{2}-\mathrm{O}(\mathrm{Me}) \mathrm{CH}(\mathrm{Me}) \mathrm{CH}_{2} \mathrm{OMe}\right]$, 2f. Colourless solid, $81 \%$ yield from 1c $(0.200 \mathrm{~g}, 0.487 \mathrm{mmol})$ and 1,2-dimethoxypropane $(0.060 \mathrm{~mL}, 0.49 \mathrm{mmol})$. Time: 24 hours. Anal. Calcd for $\mathrm{C}_{5} \mathrm{H}_{12} \mathrm{Br}_{4} \mathrm{O}_{2} \mathrm{Zr}$ : C, 11.66; H, 2.35; Zr, 17.71; Br, 62.06. Found: C, 11.73; H, 2.29; Zr, 17.64; Br, 61.39. IR (solid state): 2952w, 2846w, 1535w-m, 1447m, 1385w, 1269w, 1117w, 1066m, 1012s, 985vs, 930s, 904vs, 795s, $773 \mathrm{~s}, 699 \mathrm{~m}-\mathrm{s} \mathrm{cm}^{-1} .{ }^{1} \mathrm{H}$ NMR $\left(\mathrm{CDCl}_{3}\right): \delta=4.60 \div 4.35\left(\mathrm{~m}, 3 \mathrm{H}, \mathrm{CH}_{2} \mathrm{CH}\right), 4.23,4.11(\mathrm{~s}, 6 \mathrm{H}, \mathrm{OMe}), 1.56$ $\operatorname{ppm}\left(\mathrm{d},{ }^{3} \mathrm{~J}_{\mathrm{HH}}=6.59 \mathrm{~Hz}, 3 \mathrm{H}, \mathrm{CHMe}\right) .{ }^{13} \mathrm{C}\left\{{ }^{1} \mathrm{H}\right\} \operatorname{NMR}\left(\mathrm{CDCl}_{3}\right): \delta=82.1,80.0\left(\mathrm{CH}_{2} \mathrm{CH}\right), 69.2,64.0$ $(\mathrm{OMe}), 14.2 \mathrm{ppm}(\mathrm{CHMe}) . \Lambda_{M}=0.16 \mathrm{~S} \cdot \mathrm{cm}^{2} \mathrm{~mol}^{-1}$. Thermal treatment / hydrolysis (GC/MS-NMR): $\mathrm{CH}_{2} \mathrm{Cl}_{2}, \mathrm{MeOCH}_{2} \mathrm{CH}(\mathrm{Me}) \mathrm{OMe}$ (ratio 1:1).

$\mathrm{TiI}_{4}\left[\kappa^{2}-\mathrm{O}(\mathrm{Me}) \mathrm{CH}_{2} \mathrm{CH}_{2} \mathrm{OCH}_{2} \mathrm{Cl}\right]$, 2g. Dark red solid, $83 \%$ yield from $\mathbf{1 b}(0.210 \mathrm{~g}, 0.378 \mathrm{mmol})$ and $\mathrm{MeO}\left(\mathrm{CH}_{2}\right)_{2} \mathrm{OCH}_{2} \mathrm{Cl}(0.045 \mathrm{~mL}, 0.39 \mathrm{mmol})$. Time: 12 hours. Anal. Calcd for $\mathrm{C}_{4} \mathrm{H}_{9} \mathrm{ClI}_{4} \mathrm{O}_{2} \mathrm{Ti}$ : $\mathrm{C}, 7.06 ; \mathrm{H}$, 1.33; Ti, 7.04; I, 74.64. Found: C, 7.01; H, 1.29; Ti, 7.09; I, 74.15. ${ }^{1} \mathrm{H}$ NMR $\left(\mathrm{CDCl}_{3}\right): \delta=5.82$ (s, $2 \mathrm{H}$, $\left.\mathrm{CH}_{2} \mathrm{Cl}\right), 4.13,3.88\left(\mathrm{~m}, 4 \mathrm{H}, \mathrm{CH}_{2} \mathrm{CH}_{2}\right), 3.71 \mathrm{ppm}(\mathrm{s}, 3 \mathrm{H}, \mathrm{OMe}) .{ }^{13} \mathrm{C}\left\{{ }^{1} \mathrm{H}\right\} \mathrm{NMR}\left(\mathrm{CDCl}_{3}\right): \delta=86.1$ $\left(\mathrm{CH}_{2} \mathrm{Cl}\right), 72.7,70.6\left(\mathrm{CH}_{2} \mathrm{CH}_{2}\right), 56.8 \mathrm{ppm}(\mathrm{OMe})$. Thermal treatment / hydrolysis (GC/MS-NMR): $\mathrm{CH}_{2} \mathrm{Cl}_{2}$, MeI, $\mathrm{CH}_{2} \mathrm{ICH}_{2} \mathrm{I}, \mathrm{Me}_{2} \mathrm{O}, \mathrm{CH}_{2} \mathrm{ClI}$ (ratio 6:6:4:2:1).

$\mathrm{ZrBr}_{4}\left[\kappa^{2}-\mathrm{O}(\mathrm{Me}) \mathrm{CH}_{2} \mathrm{CH}_{2} \mathrm{OCH}_{2} \mathrm{Cl}\right], \mathbf{2 h}$. Light yellow solid, $87 \%$ yield from 1c $(0.250 \mathrm{~g}, 0.609 \mathrm{mmol})$ and $\mathrm{MeO}\left(\mathrm{CH}_{2}\right)_{2} \mathrm{OCH}_{2} \mathrm{Cl}(0.070 \mathrm{~mL}, 0.61 \mathrm{mmol})$. Time: 12 hours. Anal. Calcd for $\mathrm{C}_{4} \mathrm{H}_{9} \mathrm{Br}_{4} \mathrm{ClO}_{2} \mathrm{Zr}: \mathrm{C}, 8.97$; $\mathrm{H}, 1.69 ; \mathrm{Zr}, 17.04 ; \mathrm{Br}, 59.70$. Found: $\mathrm{C}, 9.05 ; \mathrm{H}, 1.61 ; \mathrm{Zr}, 16.91 ; \mathrm{Br}, 59.55 .{ }^{1} \mathrm{H} \mathrm{NMR}\left(\mathrm{CDCl}_{3}\right): \delta=5.82$ $\left(\mathrm{s}, 2 \mathrm{H}, \mathrm{CH} \mathrm{H}_{2} \mathrm{Cl}\right), 4.18,3.88\left(\mathrm{~m}, 4 \mathrm{H}, \mathrm{CH}_{2} \mathrm{CH}_{2}\right), 3.88 \mathrm{ppm}(\mathrm{s}, 3 \mathrm{H}, \mathrm{OMe}) .{ }^{13} \mathrm{C}\left\{{ }^{1} \mathrm{H}\right\} \mathrm{NMR}\left(\mathrm{CDCl}_{3}\right): \delta=$ $78.6\left(\mathrm{CH}_{2} \mathrm{Cl}\right), 76.0,70.8\left(\mathrm{CH}_{2} \mathrm{CH}_{2}\right), 64.8 \mathrm{ppm}(\mathrm{OMe}) . \Lambda_{M}=0.20 \mathrm{~S} \cdot \mathrm{cm}^{2} \mathrm{~mol}^{-1}$. Thermal treatment / hydrolysis (GC/MS-NMR): $\mathrm{CH}_{2} \mathrm{Cl}_{2}, \mathrm{MeBr}, \mathrm{CH}_{2} \mathrm{BrCH}_{2} \mathrm{Br}, \mathrm{O}\left(\mathrm{CH}_{2} \mathrm{CH}_{2} \mathrm{Cl}\right)_{2}, \mathrm{Me}_{2} \mathrm{O}$ (ratio: 5:10:3:2:3). 
$\mathrm{ZrI}_{4}\left[\kappa^{2}-\mathrm{O}(\mathrm{Me}) \mathrm{CH}_{2} \mathrm{CH}_{2} \mathrm{OCH}_{2} \mathrm{Cl}\right]$, 2i. Pink solid, $85 \%$ yield from 1d $(0.255 \mathrm{~g}, 0.426 \mathrm{mmol})$ and $\mathrm{MeO}\left(\mathrm{CH}_{2}\right)_{2} \mathrm{OCH}_{2} \mathrm{Cl}(0.050 \mathrm{~mL}, 0.44 \mathrm{mmol})$. Time: 12 hours. Anal. Calcd for $\mathrm{C}_{4} \mathrm{H}_{9} \mathrm{ClI}_{4} \mathrm{O}_{2} \mathrm{Zr}$ : $\mathrm{C}, 6.64$; H, 1.25; Zr, 12.61; I, 70.17. Found: C, 6.56; H, 1.21; Zr, 12.45; I, 69.66. ${ }^{1} \mathrm{H} \mathrm{NMR}\left(\mathrm{CDCl}_{3}\right): \delta=5.85(\mathrm{~s}$, $\left.2 \mathrm{H}, \mathrm{CH}_{2} \mathrm{Cl}\right), 4.31,3.87\left(\mathrm{~m}, 4 \mathrm{H}, \mathrm{CH}_{2} \mathrm{CH}_{2}\right), 3.64 \mathrm{ppm}(\mathrm{s}, 3 \mathrm{H}, \mathrm{OMe}) .{ }^{13} \mathrm{C}\left\{{ }^{1} \mathrm{H}\right\} \mathrm{NMR}\left(\mathrm{CDCl}_{3}\right): \delta=83.3$ $\left(\mathrm{CH} \mathrm{H}_{2} \mathrm{Cl}\right), 72.2,70.6\left(\mathrm{CH}_{2} \mathrm{CH}_{2}\right), 64.4 \mathrm{ppm}(\mathrm{OMe})$. Thermal treatment / hydrolysis (GC/MS-NMR): $\mathrm{CH}_{2} \mathrm{Cl}_{2}, \mathrm{MeI}, \mathrm{CH}_{2} \mathrm{ClI}, \mathrm{CH}_{2} \mathrm{ICH}_{2} \mathrm{I}$ (ratio 5:8:1:4).

\section{Acknowledgements.}

The authors wish to thank the Ministero dell'Istruzione, dell' Università e della Ricerca (MIUR, Roma), Programma di Ricerca Scientifica di Notevole Interesse Nazionale 2007-2008, for financial support.

\section{References}

[1] (a) D. Takeuchi, Dalton Trans. 39 (2010) 311;

(b) K. Nomura, Dalton Trans. (2009) 8811;

(c) E.Y.-X. Chen, Chem Rev. 109 (2009) 5157;

(d) W. Kaminsky, A. Funck, H. Hähnsen, Dalton Trans. (2009) 8803;

(d) T. Matsugi, T. Fujita, Chem. Soc. Rev. 37 (2008) 1264;

(e) W. Zhang, L.R. Sita, Adv. Synth. Catal. 350 (2008) 439;

(f) G. Fink, H.-H. Brintzinger, "Polymerization Reactions in Metal-Catalysis" in Industrial Organic Processes, Eds. G.P. Chiusoli, P.M. Maitlis, 2006, RSC Publishing, Chapter 7, p. 218. 
[2] Some recent references are: (a) V. Rodríguez-Cisterna, C. Villar, P. Romea, F. Urpí, J. Org. Chem. $72(2007) 6631$;

(b) C. Mamat, S. Büttner, T. Trabhardt, C. Fischer, P. Langer, J. Org. Chem. 72 (2007) 62;

(c) D. Basavaiah, K.R. Reddy, Org. Lett., 9 (2007) 57;

(d) V.T.H. Nguyen, E. Bellur, B. Appel, P. Langer, Synthesis (2006) 1103;

(e) L. Anastasia, E. Giannini, G. Zanoni, G. Vidari, Tetrahedron Lett. 46 (2005) 5803.

[3] G. Smitha, S. Chandrasekhar, C.S. Reddy, Synthesis (2008) 829, and references therein.

[4] (a) Y. Hayashi, M. Nakamura, S. Nakao, T. Inoue, M. Shoji, Angew. Chem., Int. Ed. 41 (2002) 4079;

(b) M. Shoji, J. Yamaguchi, H. Kakeya, H. Osada, Y. Hayashi, Angew. Chem., Int. Ed. 41 (2002) 3192.

[5] (a) F. Marchetti, G. Pampaloni, S. Zacchini, Dalton Trans. (2007) 4343;

(b) F. Marchetti, G. Pampaloni, S. Zacchini, Inorg. Chem. 47 (2008) 365;

(c) F. Marchetti, G. Pampaloni, S. Zacchini, Polyhedron 27 (2008) 1969;

(d) F. Marchetti, G. Pampaloni, S. Zacchini, Eur. J. Inorg. Chem. (2008) 453.

[6] (a) R. Bini, C. Chiappe, F. Marchetti, G. Pampaloni, S. Zacchini, Inorg. Chem. 49 (2010) 339;

(b) F. Marchetti, G. Pampaloni, S. Zacchini, Dalton Trans. (2009) 6759;

(c) F. Marchetti, G. Pampaloni, S. Zacchini, Dalton Trans. (2009) 8096;

(d) F. Marchetti, G. Pampaloni, S. Zacchini, Chem. Commun. (2008) 3651;

(e) F. Marchetti, G. Pampaloni, S. Zacchini, Dalton Trans. (2008) 7026.

[7] (a) E. Hengge, H. Zimmermann, Monatsh. Chem. 103 (1972) 418;

(b) C. Bingel, B. Schiemenz, M. Gores, Int. Patent WO99/12493 (to Hoechst Research \& Technology Deutschland), 1999; 
(c) H.-R.-H. Damrau, P. Müller, V. Garcia, C. Sidot, C. Tellier, J.-F. Lelong, Int. Patent WO2004/037840 (to Basell Polyolefine GmbH), 2004;

(d) R.J.H. Clark, W. Errington, Inorg. Chem. 5 (1966) 650;

(e) R.S. Borden, P.A. Loeffler, D.S. Dyer, Inorg. Chem. 11 (1972) 2481.

[8] (a) F. Calderazzo, G.E. De Benedetto, U. Englert, I. Ferri, G. Pampaloni, T. Wagner, Z. Naturforschg. 51B (1996) 506;

(b) E. Turin, R.M. Nielson, A.E. Merbach, Inorg. Chim. Acta 134 (1987) 67.

(c) P.A. Delaney, R.A.W. Johnstone, I.D. Entwistle, J. Chem. Soc., Perkin Trans. 1 (1986) 1855.

[9] F. Marchetti, G. Pampaloni, S. Zacchini, Polyhedron 28 (2009) 1235.

[10] (a) W.J. Evans, T.A. Ulibarri, J.W. Ziller, Organometallics 10 (1991) 134;

(b) C. Eaborn, P.B. Hitchcock, K. Izod, J.D. Smith, J. Amer. Chem. Soc. 116 (1994) 12071;

(c) B.-J. Deelman, M. Booij, A. Meetsma, J.H. Teuben, H. Kooijman, A.L. Spek, Organometallics 14 (1995) 2306;

(d) F. Preuss, G. Hornung, W. Frank, G. Reiss, S. Müller-Becker, Z. Anorg. Allg. Chem. 621 (1995) 1663;

(e) K. Takaki, M. Maruo, T. Kamata, J. Org. Chem. 61 (1996) 8332;

(f) D.J. Duncalf, P.B. Hitchcock, G.A. Lawless, Chem. Commun. (1996) 269;

(g) M.C. Cassani, M.F. Lappert, F. Laschi, Chem. Commun. (1997) 1563;

(h) C.A. Bradley, L.F. Veiros, D. Pun, E. Lobkovsky, I. Keresztes, P.J. Chirik, J. Amer. Chem. Soc. 128 (2006) 16600.

[11] (a) Y.K. Gun'ko, P.B. Hitchcock, M.F. Lappert, J. Organomet. Chem. 499 (1995) 213;

(b) M.C. Cassani, Y.K. Gun'ko, P.B. Hitchcock, A.G. Hulkes, A.V. Khvostov, M.F. Lappert, A.V. Protchenko, J. Organomet. Chem. 647 (2002) 71; 
(c) S. Le Caër, M. Heninger, J. Lemaire, P. Boissel, P. Maître, H. Mestdagh, Chem. Phys. Lett. 385 (2004) 273;

(d) S. Le Caër, M. Heninger, P. Pernot, H. Mestdagh, J. Phys. Chem. A 110 (2006) 9654;

(e) C.A. Bradley, L.F. Veiros, P.J. Chirik, Organometallics 26 (2007) 3191.

(f) I.L. Fedushkin, F. Girgsdies, H. Schumann, M.N. Bochkarev, Eur. J. Inorg. Chem. (2001) 2405.

[12] a) A. Ecker, R. Köppe, C. Üffing, H. Schnöckel, Z. Anorg. Allg. Chem. 624 (1998) 817;

b) W. Uhl, A. Vester, D. Fenske, G. Baum, J. Organomet. Chem. 464 (1994) 23;

(c) W. Uhl, R. Gerdin, A. Vester, J. Organomet. Chem. 513 (1996) 163;

(d) W. Uhl, Coord. Chem. Rev. 163 (1997) 1;

(e) M. Westerhausen, C. Birg, H. Nöth, J. Knizek, T. Seifert, Eur. J. Inorg. Chem. (1999) 2209.

[13] (a) A. Jutand, Eur. J. Inorg. Chem. (2003) 2017;

(b) W.J. Geary, Coord. Chem. Rev. 7 (1971) 81.

[14] D.A. Skoog, D.M. West, Fundamentals of Analytical Chemistry, 2nd Edition, Holt, Rinehart and Winston, Chatham, UK, 1974, p. 233. 


\section{Captions for Table}

Table 1. Summary of the organic compounds obtained from $\mathrm{MX}_{4}$ and 1,2-dialkoxyalkanes after hydrolysis of the reaction mixtures. 


\section{Scheme 1}

$\mathrm{Mx}_{4} \stackrel{\mathrm{OPO}_{\longrightarrow}}{\longrightarrow} \mathrm{x}_{4} \mathrm{M}_{-}^{-} \mathrm{O}$

\begin{tabular}{|c|c|c|c|}
\hline $\mathrm{M}$ & $\mathrm{X}$ & $\mathrm{O} O$ & \\
\hline $\mathrm{Ti}$ & $\mathrm{F}$ & dme & $\mathbf{2 a}$ \\
\hline $\mathrm{Ti}$ & I & dme & $2 \mathrm{~b}$ \\
\hline $\mathrm{Zr}$ & $\mathrm{Br}$ & dme & $2 \mathrm{c}$ \\
\hline $\mathrm{Zr}$ & I & dme & 2d \\
\hline $\mathrm{Ti}$ & I & $\mathrm{MeOCH}_{2} \mathrm{CH}(\mathrm{Me}) \mathrm{OMe}$ & $2 e$ \\
\hline $\mathrm{Zr}$ & $\mathrm{Br}$ & $\mathrm{MeOCH}_{2} \mathrm{CH}(\mathrm{Me}) \mathrm{OMe}$ & $2 f$ \\
\hline $\mathrm{Ti}$ & I & $\mathrm{MeOCH}_{2} \mathrm{CH}_{2} \mathrm{OCH}_{2} \mathrm{Cl}$ & $2 \mathrm{~g}$ \\
\hline $\mathrm{Zr}$ & $\mathrm{Br}$ & $\mathrm{MeOCH}_{2} \mathrm{CH}_{2} \mathrm{OCH}_{2} \mathrm{Cl}$ & $2 \mathrm{~h}$ \\
\hline $\mathrm{Zr}$ & I & $\mathrm{MeOCH}_{2} \mathrm{CH}_{2} \mathrm{OCH}_{2} \mathrm{Cl}$ & $2 \mathbf{i}$ \\
\hline
\end{tabular}

Table 1

\begin{tabular}{|c|c|}
\hline Compound & Organic products \\
\hline $2 a, c, d$ & dme \\
\hline $2 \mathbf{b}$ & $\mathrm{MeI}, \mathrm{CH}_{2} \mathrm{ICH}_{2} \mathrm{I}$ \\
\hline $2 \mathrm{e}$ & $\mathrm{MeI}, \mathrm{Me}_{2} \mathrm{O}$ \\
\hline $2 \mathrm{f}$ & $\mathrm{MeOCH}_{2} \mathrm{CH}(\mathrm{Me}) \mathrm{OMe}$ \\
\hline $2 \mathrm{~g}$ & $\mathrm{MeI}, \mathrm{CH}_{2} \mathrm{ICH}_{2} \mathrm{I}, \mathrm{Me}_{2} \mathrm{O}, \mathrm{CH}_{2} \mathrm{ClI}$ \\
\hline $\mathbf{2 h}$ & $\mathrm{MeBr}, \mathrm{CH}_{2} \mathrm{BrCH}_{2} \mathrm{Br}, \mathrm{O}\left(\mathrm{CH}_{2} \mathrm{CH}_{2} \mathrm{Cl}\right)_{2}, \mathrm{Me}_{2} \mathrm{O}$ \\
\hline $2 \mathbf{i}$ & $\mathrm{MeI}, \mathrm{CH}_{2} \mathrm{ICH}_{2} \mathrm{I}, \mathrm{CH}_{2} \mathrm{ClI}$ \\
\hline
\end{tabular}

